\title{
CAPACIDADES DE TI E DESEMPENHO DA FIRMA NAS EMPRESAS BRASILEIRAS MAIS INOVADORAS NO USO DA TI
}

\author{
Deyvison de Lima Oliveira \\ Doutor em Administração pelo PPGA/EA/UFRGS \\ Professor do Departamento de Ciências Contábeis da Universidade Federal de Rondônia - Campus de \\ Vilhena \\ deyvilima@gmail.com (Brasil)
}

\author{
Antonio Carlos Gastaud Maçada \\ Doutor em Administração pelo PPGA/EA/UFRGS \\ Professor do DCA/PPGA/EA/UFRGS \\ acgmacada@ea.ufrgs.br (Brasil)
}

\section{RESUMO}

O objetivo deste trabalho é identificar a (in) existência de associação direta entre capacidades de TI e desempenho no nível da firma. Para isso são utilizados dados secundários de 122 empresas mais inovadoras no uso da TI (67 delas são listadas na BM\&FBOVESPA), publicadas em ranking de revista especializada. Os indicadores de desempenho da firma são extraídos das demonstrações contábeis, tanto no sítio da Bolsa quanto nos sítios corporativos, e confrontados com o escore de capacidades de TI da empresa no ranking - abrangendo um período de três anos (2009 a 2011). Os dados são analisados com o Teste não paramétrico de Mann-Whitney, tendo em vista a ausência de normalidade dos dados. Os resultados confirmam que altas capacidades de TI estão dissociadas de melhores medidas de desempenho da firma, sinalizando para investigações que identifiquem o valor da TI em níveis inferiores ao da firma, a exemplo de processos de negócios e capacidades organizacionais que fazem uso direto da TI. Limitação e oportunidades de pesquisa são apresentadas nas conclusões.

Palavras-chave: Capacidades de TI; Desempenho da firma; Desempenho de processos; Recursos; Capacidades organizacionais. 


\section{INTRODUÇÃO}

A relação entre recursos/capacidades de TI e desempenho organizacional vem sendo estudada recentemente considerando dois aspectos decisórios centrais: (i) a decisão de uso do construto “capacidades de TI" em lugar dos recursos isolados (Hartono, Li, Na, \& Simpson, 2010; Park, Im, \& Kim, 2011); e (ii) a decisão quanto ao nível de impacto da TI - se em processos, em outras capacidades organizacionais, ou ainda, diretamente no nível de firma (G. Kim, Shin, Kim, \& Lee, 2011; Tallon, 2010).

Para o primeiro aspecto, as teorias da Visão Baseada em Recursos (Resource-Based View RBV) e Capacidades Dinâmicas são apresentadas na literatura como abordagens complementares (Lahiri, Kedia, \& Mukherjee, 2012) para identificação e compreensão do papel da TI nas organizações. A RBV entende a firma como um conjunto de recursos que, detendo certos atributos (valor, raridade, dificuldade de imitação, não possibilidade de substituição), determinam desempenho superior da firma (Barney, 1991; Kraaijenbrink, Spender, \& Groen, 2010). As Capacidades Dinâmicas defendem que esses recursos demandam reconfiguração e reorganização constantes para que garantam desempenho superior das firmas (D. J. Teece, 2007). O conceito de capacidades pressupõe também a presença de recursos, o que fundamenta a visão complementar das duas abordagens teóricas.

No tocante ao segundo aspecto, estudos recentes apontam o nível de processos como o primeiro nível de impacto da TI (Ray, Barney, \& Muhanna, 2004), a despeito de esse nível também possuir relação com o desempenho no nível da firma, como apontam alguns estudos (G. Kim et al., 2011; Qu, Oh, \& Pinsonneault, 2010). Nesta linha, a literatura tem apresentado a necessidade de investigações quanto ao impacto da TI nos diversos processos e capacidades organizacionais (Tallon, 2010), e a utilização da abordagem sobre as "capacidades de TI" - em contraposição aos recursos tecnológicos isolados (Schwarz, Kalika, Kefi, \& Schwarz, 2010).

Pesquisas abrangendo os dois aspectos decisórios fornecem respaldo inicial para a compreensão do valor das capacidades de TI em nível inferior ao nível da firma, incluindo processos e capacidades organizacionais, a despeito de estudos outros utilizarem indicadores de desempenho no nível da firma para avaliar o impacto direto da TI (Dale Stoel \& Muhanna, 2009; Huang, Ou, Chen, \& Lin, 2006). Nesta última linha, a literatura tem apresentado resultados divergentes quanto ao papel da TI nas organizações, principalmente, quando empregada a RBV para compreensão do fenômeno relacionado ao valor de negócio da TI (Masli, Richardson, Sanchez, \& Smith, 2011; Ting-Peng, JunJer, \& Chih-Chung, 2010). 
Dessa forma, atendendo às afirmações e demandas de estudos quanto ao impacto da TI em nível inferior ao da firma (G. Kim et al., 2011; Qu et al., 2010), e as divergências quanto ao impacto direto da TI no desempenho agregado da firma (Tallon, 2010; Tallon \& Kraemer, 2007), esta pesquisa reúne medidas de desempenho de empresas em um ranking de inovação no uso da TI, visando identificar a (in) existência de associação entre as capacidades de TI e indicadores de desempenho no nível da firma. Os achados permitem corroborar ou confrontar resultados anteriores dentro da abordagem incipiente quanto à utilização das capacidades de TI como construto independente (em lugar de recursos de TI) e do uso de medidas de desempenho no nível inferior ao da firma, para captar o valor da TI.

O trabalho detém os seguintes diferenciais para estudo do valor da TI: (a) utilização de medidas de desempenho da firma em quatro grupos - Endividamento, Liquidez, Variação da Receita e Rentabilidade, provenientes do campo contábil - em contraposição à predominância estrita das medidas de rentabilidade na literatura; (b) identificação da associação entre capacidades de TI e as medidas de desempenho da firma; (c) utilização de amostra de empresas com capacidades de TI comprovada por ranking profissional da área, o que permite verificar a (in) existência de associação entre altas capacidades de TI e alto nível dos indicadores; e (d) emprego de medidas de desempenho objetivas (observáveis), em contraste às medidas de percepção.

Além da introdução, o artigo está estruturado em mais quatro seções: na seção 2 apresenta-se o referencial teórico - incluindo os principais resultados da literatura na relação entre TI e desempenho, formas de associação e níveis de impactos da TI, modelo e hipóteses de pesquisa. Na seção 3 são abordados os procedimentos de coleta e análise dos dados, bem como a amostra e população da pesquisa. Na seção 4 os resultados são apresentados - abrangendo o teste das hipóteses e discussão à luz da literatura. Na última seção as conclusões e oportunidades de pesquisa são apresentadas.

\section{CAPACIDADES DE TI E DESEMPENHO}

Capacidades de TI podem ser conceituadas como "habilidade de mobilizar e organizar TI - que representa os recursos baseados em combinação ou co-presença com outros recursos e capacidades" (Z. Wu, Huang, \& Wu, 2008, p. p. 526). As capacidades de TI também são vistas como a habilidade da firma em reunir, integrar e desenvolver recursos baseados em TI (Liu, Lu, \& Hu, 2008).

Assim, essas capacidades são parte das capacidades da firma, e são de difícil replicação por concorrentes (Liu et al., 2008; F. Wu, Yeniyurt, Kim, \& Cavusgil, 2006), tendo em vista a conexão delas com a história, cultura e experiência da firma (A. Bharadwaj, Sambamurthy, \& Zmud, 1999). 
Essa visão das capacidades de TI é fundamentada pela Visão Baseada em Recursos (ResourceBased View - RBV) - que relaciona recursos/capacidades valiosos, raros, difíceis de imitar e insubstituíveis com o melhor desempenho da firma (Barney, 1991, 2001; Kraaijenbrink et al., 2010). É corroborada também pela Teoria das Capacidades Dinâmicas (Theory of Dynamic Capabilities TDC), que enfatiza a importância das capacidades de reconfiguração da base de recursos para uso da firma - a fim de garantir vantagem competitiva (Barreto, 2010; Silva, 2011; David J. Teece, Pisano, \& Shuen, 1997).

As seções seguintes discutem a relação entre capacidades de TI e desempenho considerando as teorias em questão e alguns aspectos dessa relação, a saber: formas de associação entre TI e desempenho e nível de desempenho (processos, capacidades, firma).

\subsection{Associação entre capacidades de TI e desempenho: direta versus indireta}

No conjunto das investigações da literatura, as capacidades de TI são apresentadas relacionando-se com desempenho de formas variadas. Alguns estudos defendem o relacionamento direto (Huang et al., 2006; Santhanam \& Hartono, 2003; Tian, Wang, Chen, \& Johansson, 2010). Nesta linha, Masli et al. (2011) identificaram relação positiva entre capacidades de TI superior e desempenho no nível da firma no período de 1988 a 2007 - embora destaquem a tendência na redução do impacto da capacidade de TI no desempenho a partir de 1999, justificada pela crise nas empresas “ponto.com” e pela vida curta da vantagem competitiva dirigida por TI (Masli et al., 2011).

Outros estudos defendem o impacto da TI no desempenho mediado por demais construtos, como tomada de decisão (WU, HUANG e WU, 2008) e capacidades dinâmicas orientadas a processos (G. Kim et al., 2011). Contudo - considerando que as capacidades dinâmicas afetam em primeira mão a base de recursos, e então, o desempenho (Ambrosini, Bowman, \& Collier, 2009) - essa teoria defende que há uma lacuna (variável, construto) entre as capacidades de TI e desempenho, que seria representada por uma base de recurso da organização ou capacidades organizacionais. Portanto, para as Capacidades Dinâmicas a relação direta entre capacidades e desempenho da firma é não factível.

\subsection{Níveis de desempenho da TI: firma versus processos/capacidades}

Além das discussões sobre a forma de impacto da TI e os possíveis mediadores, estudos recentes têm defendido abordagem alternativa para o nível de impacto da TI (G. Kim et al., 2011; Silva, 2011). A análise do impacto no nível de firma (medidas agregadas como retorno sobre 
investimento, retorno sobre ativo, margem de lucro etc.) tem cedido espaço para a análise de impacto no nível de processos (por exemplo: relação com clientes e fornecedores, marketing, tomada de decisão, produção e operações, melhoria do produto) (Lucht, Hoppen, \& Maçada, 2007; Ray et al., 2004; Tallon, 2010; Tallon \& Kraemer, 2007) - com base na afirmação de que o desempenho no nível da firma é resultante de um conjunto de variáveis (Goldoni \& Oliveira, 2010; Ting-Peng et al., 2010), da qual a TI é parte.

De igual forma, investigações no campo da Visão Baseada em Recursos (RBV) e das Capacidades Dinâmicas (TDC) têm compartilhado indicações para uso de medidas de desempenho em nível inferior ao da firma (Ray et al., 2004; Ray, Muhanna, \& Barney, 2005). Isso porque a TI tende ao impacto inicial nos processos organizacionais (Silva, 2011), para posterior impacto nas medidas financeiras no nível da firma (G. Kim et al., 2011).

Seguindo as premissas da RBV, a literatura evidencia o impacto dos recursos de TI no desempenho, por meio das capacidades organizacionais que aqueles recursos alavancam (Ting-Peng $e t$ al., 2010).

Como visão alternativa e avanço à RBV, que defende a importância de recursos únicos para o desempenho da firma, as Capacidades Dinâmicas defendem que a base de recursos que gera desempenho demanda reconfiguração constante (AMBROSINI e BOWMAN, 2009; BARRETO, 2010). Além disso, os recursos por si sós não gerariam vantagem competitiva e desempenho, tendo em vista o aspecto estático da base de ativos (WU, 2010). Assim, por esta abordagem, há associação indireta entre capacidades dinâmicas e desempenho, por meio da base de recursos da firma (AMBROSINI e BOWMAN, 2009) ou mediante outras capacidades organizacionais.

Além de ser "possuído", como defende a RBV, o recurso precisa ser estrategicamente gerenciado e desenvolvido, a fim de gerar desempenho superior - sendo a capacidade de gestão uma variável moderadora entre capacidades de TI e desempenho da firma (Lahiri et al., 2012) - o que fundamenta o papel das capacidades de TI como suporte a processos e a outras capacidades organizacionais antes de qualquer impacto no desempenho agregado da firma.

Com base na forma de associação entre capacidades de TI e desempenho, discutida na literatura, bem como os diferentes níveis de desempenho abordados, um modelo conceitual emerge como representativo da discussão apresentada - como traz a Figura 1. 
Figura 1 - Modelo conceitual: formas de associação e níveis de desempenho.

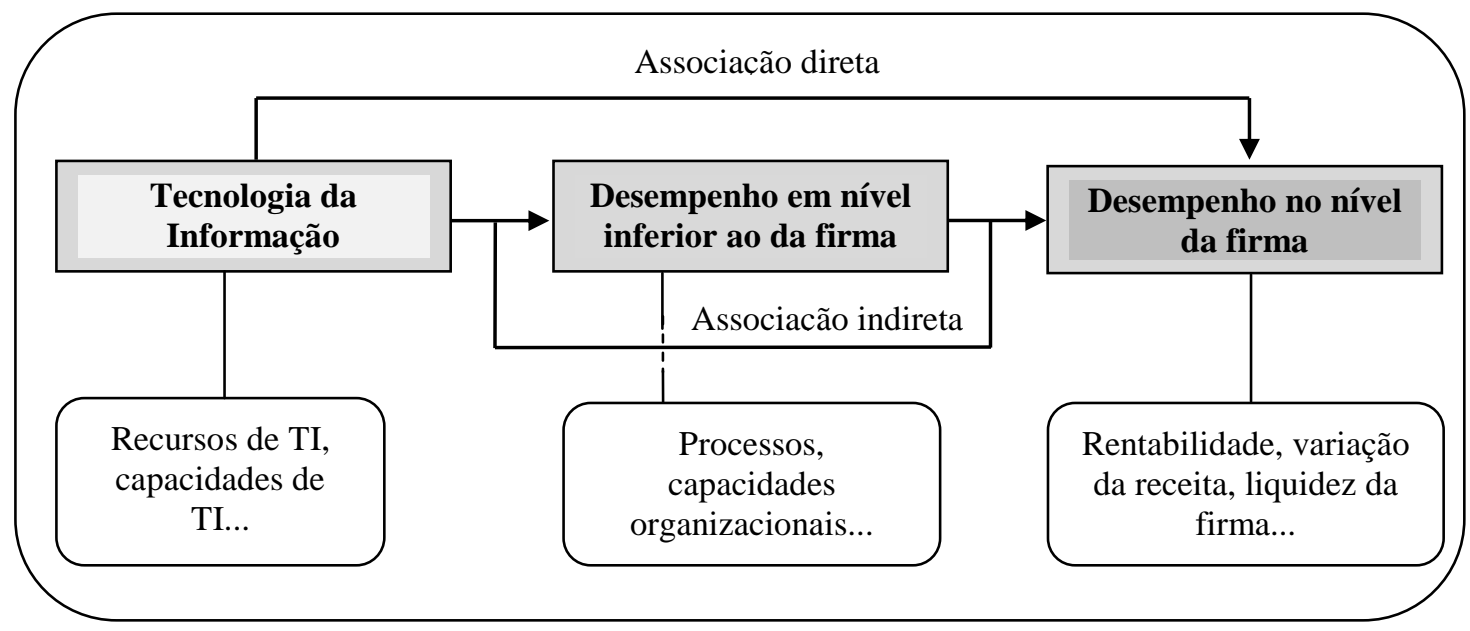

Fonte: Adaptado da literatura.

\subsection{Capacidades de TI $x$ desempenho: síntese das associações}

Considerando a revisão de literatura das seções anteriores, um resumo dos resultados da relação entre "capacidades de TI e desempenho" é apresentado no Quadro 1 - contemplando os autores, os resultados significativos e teorias de base utilizadas. No resumo constam tanto os resultados de estudos que consideraram o nível de desempenho de processos quanto o nível da firma, para avaliação do valor da TI.

Quadro 1 - Resultados da relação “capacidades de TI - desempenho".

\begin{tabular}{|c|c|c|}
\hline Autores & Resultados relacionados (significância estatística dos links) & Teorias de base \\
\hline $\begin{array}{l}\text { Santhanam e Hartono } \\
\qquad(2003)\end{array}$ & Capacidades de TI $\rightarrow$ desempenho da firma & RBV \\
\hline Huang et al. (2006) & Ativos intangíveis ligados a TI $\rightarrow$ desempenho da firma & $\mathrm{RBV}$ \\
\hline $\begin{array}{l}\text { Tallon e Kraemer } \\
\qquad(2007)\end{array}$ & $\begin{array}{l}\text { Impactos da TI ao nível de processos } \rightarrow \text { impactos da TI ao nível } \\
\text { da firma }\end{array}$ & $\begin{array}{l}\text { Teoria do } \\
\text { Sensemaking }\end{array}$ \\
\hline $\begin{array}{l}\text { Wu, Huang e Wu } \\
\qquad(2008)\end{array}$ & $\begin{array}{l}\text { Capacidades de TI para exploitation } \rightarrow \text { tomada de decisão } \\
\text { operacional } \rightarrow \text { desempenho da firma; } \\
\text { Capacidades de TI para exploration } \rightarrow \text { tomada de decisão } \\
\text { gerencial } \rightarrow \text { desempenho da firma. }\end{array}$ & RBV \\
\hline $\begin{array}{l}\text { Stoel e Muhanna } \\
\text { (2009) }\end{array}$ & $\begin{array}{l}\text { Capacidades de TI focadas internamente } \rightarrow \text { desempenho da firma; } \\
\text { Capacidades de TI focadas } \text { externamente } \rightarrow \text { desempenho da firma. }\end{array}$ & RBV \\
\hline Hartono et al. (2010) & $\begin{array}{l}\text { Capacidades de infra-estrutura de TI } \rightarrow \text { qualidade da informação } \\
\text { compartilhada desempertho da firma }\end{array}$ & S/T (Sem Teoria) \\
\hline $\begin{array}{c}\text { Qu, Oh e } \\
\text { Pinsonneault (2010) }\end{array}$ & $\begin{array}{l}\text { Oferta de TI internamente } \rightarrow \text { processos de negócios ligados à TI } \\
\text { destmpenho da firma }\end{array}$ & $\begin{array}{l}\text { Visão Baseada em } \\
\text { Conhecimento }\end{array}$ \\
\hline Kim et al. (2011) & $\begin{array}{l}\text { Expertise do pessoal de TI } \rightarrow \text { capacidades de gestão de } \mathrm{TI} \rightarrow \\
\text { Flexibilidade de infra-estrutura de } \mathrm{TI} \rightarrow \text { capacidades dinâmicas } \\
\text { orientadas a processos } \quad \text { desempho da firma }\end{array}$ & TDC e RBV \\
\hline
\end{tabular}

Fonte: Elaborado a partir da literatura. 
Observa-se pelos resultados (Quadro 1) que estudos mais recentes têm a preocupação de considerarem construtos intermediários entre "capacidades de TI e desempenho da firma", sejam bases de recursos ou capacidades organizacionais (Hartono et al., 2010; G. Kim et al., 2011), sejam processos organizacionais (Qu et al., 2010; Z. Wu et al., 2008).

Tendo em vista resultados divergentes quanto ao nível (e forma) de impacto da TI (Ting-Peng et al., 2010), o contraste entre medidas de desempenho no nível da firma e o grau de capacidades de TI tende a contribuir com o entendimento do nível de impacto da TI - se no desempenho agregado ou em níveis inferiores (como processos ou capacidades organizacionais). O primeiro passo é confirmar a (in) existência de associação entre capacidades de TI e medidas de desempenho da firma - o que é proposto neste estudo.

Assim, a partir dos resultados da literatura e oportunidades de investigação, um modelo de pesquisa é apresentado na seção 2.4 , onde constam capacidades de TI e os indicadores de desempenho da firma.

\subsection{Modelo de pesquisa: hipóteses e variáveis}

O modelo de pesquisa da Figura 2 inclui dois grupos de variáveis: (i) independente capacidades de TI; (ii) dependentes: medidas de desempenho no nível da firma, agrupadas em medidas de endividamento, liquidez, variação da receita e rentabilidade.

\subsubsection{Hipóteses de pesquisa}

Considerando os resultados da literatura revisada quanto ao impacto da TI primeiramente no nível de processos (Tallon, 2010; Z. Wu et al., 2008) e capacidades organizacionais (G. Kim et al., 2011), bem como os resultados divergentes quanto ao impacto direto dos recursos e capacidades de TI no nível da firma (Masli et al., 2011; Ting-Peng et al., 2010), tem-se a hipótese de pesquisa:

H1: A associação direta entre capacidades de TI e as medidas de desempenho agregado no nível da firma é não significativa para os seguintes grupos de medidas de desempenho:

(a) Endividamento;

(b) Liquidez;

(c) Variação da receita; e

(d) Rentabilidade. 
Figura 2 - Modelo e hipóteses de pesquisa

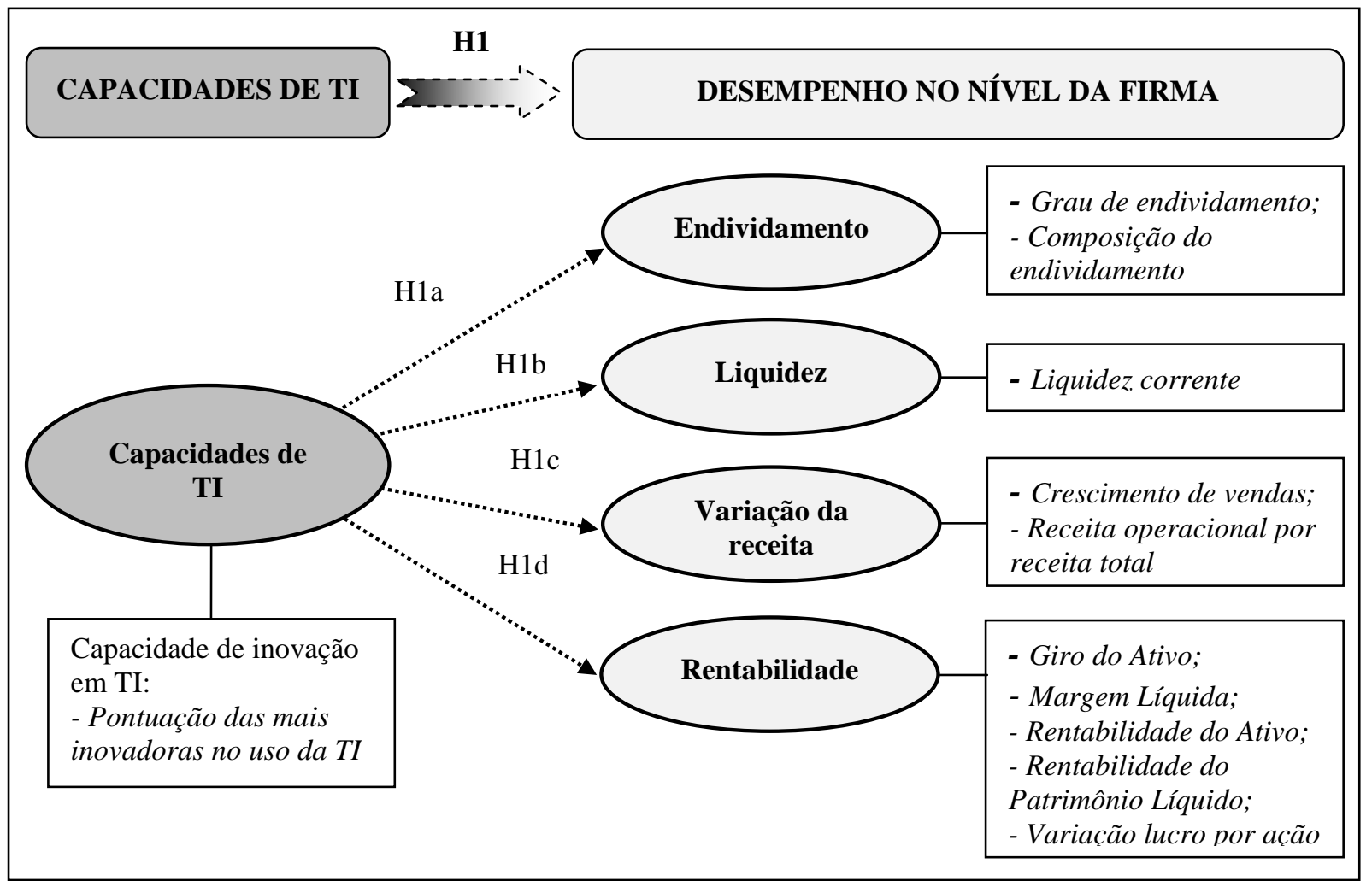

Fonte: Elaborado a partir da literatura.

\subsubsection{Descrição das variáveis}

Capacidades de TI. Na perspectiva dos dados primários, os estudos que consideram as diferentes facetas das capacidades de TI as mensuram com base em questionários de pesquisa survey, utilizando-se de escalas Likert na maioria dos casos (por exemplo: Hartono et al., 2010; Park et al., 2011). Contudo, esta pesquisa parte de dados secundários, em especial, quanto à variável “Capacidades de TI”. Utiliza a pontuação da Revista InformationWeek (ItWeb, 2009-2011) como substituto para as medidas de percepção das facetas de Capacidades de TI e segue, portanto, abordagem metodológica empregada em estudos clássicos de impactos da TI (como: A. S. Bharadwaj, 2000; Muhanna \& Stoel, 2010; Santhanam \& Hartono, 2003). No tocante ao teste de teorias, Stoel e Muhanna (2009) entendem que o uso de dados secundários para essa finalidade é bem aceito.

Desempenho no nível da firma. As investigações no campo dos impactos da TI, que analisam desempenho no nível da firma, comumente adotam as seguintes medidas: retorno sobre investimentos e sobre o patrimônio dos acionistas, margem de lucro (percentual) e lucro por ação (Huang et al., 2006; J. K. Kim, Xiang, \& Lee, 2009; Masli et al., 2011). Uma medida não empregada comumente nos estudos de TI, mas indicada pela literatura contábil é o giro do ativo (Matarazzo, 2003) - que também foi incorporada nesta pesquisa como variável de desempenho da firma. Outras cinco medidas são 
extraídas da literatura de análise de demonstrações contábeis e estão associadas ao desempenho da gestão de ativos e passivos, a saber: (a) estrutura de capital (endividamento, composição do endividamento); (b) liquidez (liquidez corrente); e (c) variação de receita (crescimento de vendas, participação da receita operacional na receita total) (Iudícibus, 2008; Matarazzo, 2003).

As medidas de desempenho referem-se ao ano anterior à pontuação das empresas na Revista, como procedido por estudos prévios (Santhanam \& Hartono, 2003; Stoel \& Muhanna, 2009).

\section{MÉTODO}

Os procedimentos de coleta e análise dos dados, bem como a amostra e população da pesquisa são descritos nesta seção.

\subsection{Amostra e coleta de dados}

A população de pesquisa compreende as 100 empresas mais inovadoras no uso da TI no Brasil avaliadas anualmente pela Revista InformationWeek (ItWeb, 2009-2011), no período de 2009 a 2011. Para a amostra de pesquisa adotaram-se dois critérios para seleção das empresas em cada período de análise: (a) empresas constantes na lista da Revista e que também estavam listadas na BM\&FBOVESPA (BM\&FBOVESPA, 2012); e (b) empresas na lista da Revista, não listadas na Bolsa, mas que possuíam as demonstrações contábeis acessíveis em seus sítios corporativos.

O primeiro critério (empresas listadas na Bovespa) foi definido em virtude da certeza quanto à disponibilidade das demonstrações contábeis dessas empresas, tendo em vista a obrigatoriedade da publicação das informações conforme a legislação vigente. A partir da identificação dessas empresas, as demonstrações financeiras foram acessadas e as medidas (desempenho da firma) foram coletadas para a base de dados.

O segundo critério (empresas não listadas, mas com demonstrações na web) se justifica também pela necessidade de acesso aos indicadores econômico-financeiros para o desempenho da firma. As demonstrações contábeis dessas empresas foram encontradas nos respectivos sítios corporativos, a partir de ferramentas de busca eletrônica (como o Google, 2012).

Da amostra original de 125 empresas, três foram excluídas por não apresentarem dados para uma ou mais variáveis (a exemplo da variável "lucro por ação ou cota").

Considerando esse refinamento, consta na Tabela 1 o total das empresas listadas na InformationWeek (população) nos anos de 2009 a 2011 e a amostra final de pesquisa - empresas listadas e não listadas na bolsa.

Revista de Administração e Inovação, São Paulo, v. 10, n.1, p. 79-97, Jan./Mar. 2013. 
Tabela 1 - Amostra de empresas em relação à Bolsa

\begin{tabular}{c|c|c|c|c}
\hline \multirow{2}{*}{ Ano } & \multicolumn{2}{|c|}{ Amostra } & \multirow{2}{*}{$\begin{array}{c}\text { Amostra } \\
\text { Total }\end{array}$} & \multirow{2}{*}{ População } \\
\cline { 2 - 3 } & Listadas & Não listadas & 31 & 100 \\
2009 & 19 & 12 & 46 & 100 \\
2010 & 28 & 18 & 45 & 100 \\
\hline Total & 20 & 25 & $\mathbf{1 2 2}$ & $\mathbf{3 0 0}$ \\
\hline
\end{tabular}

Fonte: Dados da pesquisa.

\subsection{Procedimentos para análise dos dados}

A técnica utilizada para análise estatística dos dados é o teste de Mann-Whitney, considerando que as variáveis envolvidas no estudo não atendem ao pressuposto da normalidade dos dados (Field, 2009) - o que pode ser justificado pelas diferenças nas características intrínsecas das empresas (ramos de atuação, estrutura organizacional, contextos operacionais e estratégicos, volumes de transação, setor de regulação etc.).

Pelo teste de Mann-Whitney, as observações da variável dependente (desempenho da firma) são ordenadas em postos (1 para a menor observação, 2 para a segunda menor e assim por diante). A variável independente (neste caso, Capacidades de TI), sendo categórica, é associada à dependente em conformidade com os postos. O teste, portanto, mensura a diferença de médias para os indicadores de desempenho considerando a categorização da variável independente. Em suma, o teste de MannWhitney permite verificar se há associação entre altas capacidades de TI e altos indicadores de desempenho, ou baixas capacidades de TI e baixo desempenho.

Portanto, para cada variável dependente do estudo aplica-se teste de Mann-Whitney com a variável independente "capacidades de TI". Essa variável é categorizada de acordo com seus escores do ranking mencionado, em "baixas capacidades de TI" e "altas capacidades de TI". A amostra é posta em ordem crescente, sendo que a primeira metade das observações é denominada "baixas capacidades de TI"; a segunda metade é denominada "altas capacidades de TI" - totalizando 61 observações em cada grupo. 


\section{RESULTADOS E DISCUSSÃO}

Nesta seção são caracterizadas as empresas da amostra (com base nos atributos receita líquida, setor da indústria e tempo de atuação), as hipóteses de pesquisa são testadas e os resultados são discutidos com base no referencial da seção 2. O software estatístico "Statistical Package for the Social Sciences" (IBM SPSS) foi usado para análise dos dados.

A Tabela 2 apresenta a distribuição das empresas da amostra de acordo com suas principais características.

Tabela 2 - Caracterização da amostra.

\begin{tabular}{l|c|c}
\hline \multicolumn{1}{c|}{ Características } & n & Percentual $(\%)$ \\
\hline Receita líquida anual $(\boldsymbol{R} \$)$ & - & - \\
\hline De 40 a 999 milhões & 34 & 27,9 \\
De 1 a 4 bilhões & 48 & 39,3 \\
De 4,001 a 10 bilhões & 18 & 14,8 \\
Acima de 10 bilhões & 22 & 18,0 \\
\hline Setor da indústria & - & - \\
\hline Comércio & 09 & 07,4 \\
Produção & 49 & 40,2 \\
Serviços & 64 & 52,4 \\
\hline Idade (tempo de atuação) & - & - \\
\hline 0 a 15 anos & 16 & 13,1 \\
16 a 40 anos & 34 & 27,9 \\
41 a 80 anos & 48 & 39,3 \\
Mais de 80 anos & 24 & 19,7 \\
\hline Lucro líquido médio =2,3 bilhões $(\boldsymbol{R} \$)$ & - & - \\
\hline Total & $\mathbf{1 2 2}$ & $\mathbf{1 0 0}$ \\
\hline
\end{tabular}

Fonte: Dados da pesquisa.

\subsection{Testes das hipóteses}

Para teste das hipóteses, toma-se cada uma das variáveis dependentes (medidas de desempenho) e compara-se o escore do seu posto com a categoria da variável independente. O objetivo é verificar se altas capacidades de TI estão associadas aos maiores escores dos indicadores de desempenho.

Antes de testar as hipóteses, o Teste de Kolmogorov-Smirnov (K-S) para verificação da normalidade dos dados é apresentado na Tabela 3. 
Capacidades de TI e Desempenho da Firma nas Empresas Brasileiras mais Inovadoras no uso da TI

Tabela 3 - Testes de Normalidade

\begin{tabular}{l|r|r|r|r|r|r}
\hline \multirow{2}{*}{ Variáveis } & \multicolumn{3}{|c|}{ Kolmogorov-Smirnov $^{\mathrm{a}}$} & \multicolumn{3}{|c}{ Shapiro-Wilk } \\
\cline { 2 - 7 } & Estatística & \multicolumn{1}{c|}{$\mathrm{gl}$} & \multicolumn{1}{c}{ Sig. } & Estatística & gl & \multicolumn{1}{c}{ Sig. } \\
\hline Endividamento & 0,292 & 122 & $\mathbf{0 , 0 0 0}$ & 0,660 & 122 & 0,000 \\
CompEndivi & 0,070 & 122 & $\mathbf{0 , 2 0 0}$ & 0,966 & 122 & 0,004 \\
LiqCor & 0,296 & 122 & $\mathbf{0 , 0 0 0}$ & 0,499 & 122 & 0,000 \\
CrescVendas & 0,278 & 122 & $\mathbf{0 , 0 0 0}$ & 0,374 & 122 & 0,000 \\
\%RecOpe & 0,175 & 122 & $\mathbf{0 , 0 0 0}$ & 0,778 & 122 & 0,000 \\
GirAtivo & 0,162 & 122 & $\mathbf{0 , 0 0 0}$ & 0,842 & 122 & 0,000 \\
MargLíq & 0,272 & 122 & $\mathbf{0 , 0 0 0}$ & 0,417 & 122 & 0,000 \\
RentAtivo & 0,167 & 122 & $\mathbf{0 , 0 0 0}$ & 0,726 & 122 & 0,000 \\
RentPL & 0,256 & 122 & $\mathbf{0 , 0 0 0}$ & 0,698 & 122 & 0,000 \\
VarLucAção & 0,486 & 122 & $\mathbf{0 , 0 0 0}$ & 0,213 & 122 & 0,000 \\
\hline
\end{tabular}

a. Correção de significância Lilliefors. *. Este é o menor limite da significância verdadeira.

Fonte: Dados da pesquisa.

Do conjunto de 10 variáveis de desempenho da firma, apenas uma (crescimento de vendas) apresenta distribuição normal de acordo com o teste K-S. Essa condição justifica o uso do Teste de Mann-Whitney para verificação das hipóteses, visto que é um dos testes não-paramétricos disponíveis para amostras independentes (neste caso, observações/empresas diferentes).

Dessa forma, a Tabela 4 apresenta o teste de Mann-Whitney, que compara o nível de capacidades de TI (altas x baixas) com as 10 variáveis de desempenho da firma, no intuito de verificar se altas capacidades de TI estão associadas aos altos indicadores de desempenho da firma.

Para amostras grandes (maiores que 100) e com dados que não seguem a distribuição normal, o método de estimação da significância a utilizar para interpretação dos resultados é o Método de Monte Carlo - considerando que é mais preciso que os outros (por exemplo: Métodos Exato e Assintótico) nessas situações (Field, 2009). 
Tabela 4 - Testes Estatísticos ${ }^{\mathrm{b}}$ de Mann-Whitney

\begin{tabular}{|c|c|c|c|c|c|c|c|c|c|c|c|c|}
\hline \multicolumn{3}{|c|}{ Estatísticas } & $\begin{array}{l}\text { Endivi- } \\
\text { damento }\end{array}$ & $\begin{array}{l}\text { Comp. } \\
\text { Endivi }\end{array}$ & LiqCor & $\begin{array}{l}\text { Cresc. } \\
\text { Vendas }\end{array}$ & $\begin{array}{l}\text { \%Rec. } \\
\text { Ope }\end{array}$ & $\begin{array}{l}\text { Gir. } \\
\text { Ativo }\end{array}$ & MargLíq & $\begin{array}{l}\text { Rent. } \\
\text { Ativo }\end{array}$ & RentPL & $\begin{array}{l}\text { VarLuc. } \\
\text { Ação }\end{array}$ \\
\hline \multicolumn{3}{|l|}{ Mann-Whitney U } & 1725,50 & 1707,50 & 1724,50 & 1534,50 & 1709,50 & 1655,50 & 1791,50 & 1714,50 & 1805,50 & 1677,50 \\
\hline \multicolumn{3}{|l|}{ Wilcoxon W } & 3616,50 & 3598,50 & 3615,50 & 3425,50 & 3600,50 & 3546,50 & 3682,50 & 3605,50 & 3696,50 & 3568,50 \\
\hline \multicolumn{3}{|l|}{$\mathrm{Z}$} & $-0,691$ & $-0,783$ & $-0,696$ & $-1,669$ & $-0,773$ & $-1,050$ & $-0,353$ & $-0,748$ & $-0,282$ & $-0,937$ \\
\hline \multicolumn{3}{|l|}{ Asymp. Sig. (2-tailed) } & 0,489 & 0,433 & 0,486 & 0,095 & 0,439 & 0,294 & 0,724 & 0,455 & 0,778 & 0,349 \\
\hline \multirow[t]{3}{*}{ Monte Carlo Sig. (2-tailed) } & Sig. & & $0,485^{\mathrm{a}}$ & $0,428^{\mathrm{a}}$ & $0,475^{\mathrm{a}}$ & $0,094^{\mathrm{a}}$ & $0,439^{\mathrm{a}}$ & $0,289^{\mathrm{a}}$ & $0,719^{\mathrm{a}}$ & $0,450^{\mathrm{a}}$ & $0,781^{\mathrm{a}}$ & $0,344^{\mathrm{a}}$ \\
\hline & $\begin{array}{l}\text { 99\% Confidence } \\
\text { Interval }\end{array}$ & Lower Bound & 0,472 & 0,415 & 0,462 & 0,087 & 0,427 & 0,277 & 0,708 & 0,438 & 0,770 & 0,332 \\
\hline & & Upper Bound & 0,497 & 0,440 & 0,488 & 0,102 & 0,452 & 0,300 & 0,731 & 0,463 & 0,791 & 0,356 \\
\hline \multirow[t]{3}{*}{ Monte Carlo Sig. (1-tailed) } & & Sig. & $0,240^{\mathrm{a}}$ & $0,214^{\mathrm{a}}$ & $0,237^{\mathrm{a}}$ & $0,050^{\mathrm{a}}$ & $0,220^{\mathrm{a}}$ & $0,143^{\mathrm{a}}$ & $0,367^{\mathrm{a}}$ & $0,223^{\mathrm{a}}$ & $0,394^{\mathrm{a}}$ & $0,173^{\mathrm{a}}$ \\
\hline & $\begin{array}{l}99 \% \text { Confidence } \\
\text { Interval }\end{array}$ & Lower Bound & 0,229 & 0,204 & 0,226 & 0,044 & 0,210 & 0,134 & 0,355 & 0,212 & 0,382 & 0,163 \\
\hline & & Upper Bound & 0,251 & 0,225 & 0,248 & 0,055 & 0,231 & 0,152 & 0,379 & 0,234 & 0,407 & 0,182 \\
\hline
\end{tabular}

a. Baseado em 10.000 tabelas amostrais com início de semente 2.000.000.

b. Variável de agrupamento: Capacidades de TI (altas x baixas).

Fonte: Dados da pesquisa

\subsection{Discussão}

Como podem ser observadas na Tabela 4, todas as hipóteses de pesquisa (H1a, H1b, H1c e H1d) são confirmadas. Portanto, os níveis de capacidades de TI (altas x baixas) não estão associados com as medidas de desempenho no nível da firma, para a amostra em questão.

Analisando detidamente os resultados para cada variável dependente em confronto com a medida de capacidades de TI, é possível compreender o significado da dissociação identificada.

Para os indicadores de endividamento (grau de endividamento e composição do endividamento) a interpretação latente dos resultados permite considerar que empresas com altas capacidades de TI não apresentam indicadores de endividamento melhores que as empresas com capacidades de TI baixas (H1a).

No tocante à liquidez, compreensão similar é extraída: as empresas analisadas não possuem melhor liquidez corrente quando detém altas capacidades de TI (H1b). O crescimento de vendas e a participação da receita operacional sobre a receita total, de igual forma, não estão associados ao nível de capacidades de TI (H1c).

O quarto grupo de indicadores de desempenho é o mais empregado na literatura de TI para mensurar desempenho da firma (Masli et al., 2011; Muhanna \& Stoel, 2010). Contudo, na mesma linha das hipóteses anteriores, os resultados indicam que altas medidas de rentabilidade não estão associadas às capacidades de TI altas (H1d). 
Os resultados não significativos da associação entre capacidades de TI e medidas de desempenho no nível da firma evidenciam que a influência das capacidades de TI não pode ser medida diretamente nesse nível - o que pressupõe a existência de variáveis e construtos intermediários.

Nesta linha, esses resultados são coerentes com os estudos que identificaram o valor da TI primeiramente no nível de processos (Tallon, 2010; Z. Wu et al., 2008). Estes estudos defendem que a primeira contribuição da TI está no nível de processos organizacionais diversos, dentre eles: processos inter-organizacionais (relações com fornecedores, relações com clientes, marketing e vendas), intraorganizacionais (produção e operações, melhoria de produto e serviço) (Tallon \& Kraemer, 2007) e tomada de decisão (Lucht et al., 2007).

Os resultados também corroboram outro grupo de resultados da literatura afim, o daqueles que defendem que as capacidades de TI exercem impacto em outras capacidades organizacionais (G. Kim et al., 2011; Qu et al., 2010) e em outros recursos (Hartono et al., 2010) para, posteriormente, impactarem o desempenho da firma. Portanto, o impacto indireto da TI no desempenho da firma (em contraste ao impacto direto) é defendido nesses estudos.

Esta última abordagem está em sintonia com as RBV e as Capacidades Dinâmicas. Considerando que essas teorias são visões complementares sobre o papel de recursos e capacidades no desempenho da firma, observa-se que os resultados corroboram as premissas de ambas.

Quanto à RBV, destaca-se que a não associação direta entre capacidades de TI e desempenho da firma permite o entendimento de que existem recursos (valiosos, raros, difíceis de imitar e não substituíveis) em que a TI agrega valor. Esses recursos precisam ser levantados e testados à luz da RBV (Nevo \& Wade, 2008).

Por outro lado, os resultados corroboram as premissas das Capacidades Dinâmicas no tocante à lacuna que permanece entre capacidades de TI e medidas de desempenho. Tal espaço - conforme resultados anteriores - é preenchido por outras capacidades organizacionais alavancadas pela TI (Ambrosini et al., 2009; D. J. Teece, 2007).

Como implicações estratégicas e gerenciais dos resultados da pesquisa, algumas são evidenciadas: i) a demanda por construção de instrumentos gerenciais que mensurem o valor da TI para o negócio em níveis intermediários ao nível da firma, a exemplo de processos de negócios e capacidades organizacionais; ii) a captação do valor indireto da TI para o desempenho da firma passa a considerar variáveis mediadoras que recebem o impacto de recursos e capacidades de TI, mas não representam a TI propriamente dita - no caso dos processos de negócios (relação com clientes e fornecedores, vendas e marketing, melhoria do produto/serviço, produções e operações etc.) e as diversas capacidades organizacionais que fazem uso da TI (capacidades de gestão, de comércio 
eletrônico, de compartilhamento de informação na cadeia de suprimentos etc.); iii) os efeitos dos investimentos em TI, de igual forma, tendem a ser visíveis (mensurados) primeiramente nos processos de negócios, em contraste às tentativas gerenciais de mensurar esse valor da TI diretamente no nível da firma.

\section{CONCLUSÕES E RECOMENDAÇÕES DE PESQUISA}

A utilização apenas de empresas em que os dados de desempenho da firma estavam disponíveis implica na inserção de observações com características diferenciadas na amostra, o que pode contribuir com oscilações nos resultados, considerando a premissa de que o papel e foco da TI podem ser diferenciados, a depender do cenário de atuação e demais aspectos organizacionais. Contudo, essa limitação é reduzida, considerando que o valor da TI é fenômeno comum em todos os cenários de negócios, variando apenas a intensidade desse valor - o que permite a identificação do fenômeno nos mais diversos cenários.

O objetivo da pesquisa foi o de identificar a (in) existência de associação direta entre as capacidades de TI e indicadores de desempenho no nível da firma.

As quatro hipóteses enunciadas (H1a, H1b, H1c e H1d) foram confirmadas, indicando que as capacidades de TI não estão associadas às medidas de desempenho da firma. Esses resultados representam que o valor da TI não é captado diretamente no nível da firma e que, portanto, existem outras variáveis que mensuram o desempenho direto da TI - que não as medidas da firma.

Consideração similar é que os resultados sinalizam para o impacto da TI em nível inferior ao da firma, dentre esses níveis estão os processos e capacidades organizacionais apontados pela literatura recente. Um desdobramento desta conclusão é a existência de outras variáveis organizacionais que contribuem, direta ou indiretamente, com o desempenho da firma.

O pressuposto deste estudo é que a identificação do valor da TI passa pela identificação de variáveis que não mensurem esse impacto diretamente, a exemplo de estudos que buscaram encontrar a associação direta entre a TI e medidas de rentabilidade (G. Kim et al., 2011; Tallon \& Kraemer, 2007) em algumas de suas hipóteses - e constaram não significantes.

A ausência de associação entre capacidades de TI e medidas de desempenho da firma, bem como os resultados que associam o valor da TI diretamente a essas medidas de desempenho (Masli et al., 2011; Muhanna \& Stoel, 2010), levantam algumas oportunidades de pesquisa, a saber: (i) o teste de outras variáveis (e construtos) em níveis inferiores ao da firma, a exemplo, das diversas capacidades organizacionais que podem ser alavancadas pela TI; (ii) o teste de variáveis mediadoras entre capacidades de TI e desempenho da firma, como as bases de recursos apoiadas pela TI; e (iii) a 
aplicação deste estudo com empresas de menor porte (micro, pequeno e médio), visto que a amostra constou, predominantemente, de empresas de grande porte.

\section{REFERÊNCIAS}

Ambrosini, V., Bowman, C., \& Collier, N. (2009). Dynamic capabilities: an exploration of how firms renew their resource base. [Article]. British Journal of Management, 20, S9-S24. doi:10.1111/j.1467-8551.2008.00610.x

Barney, J. B. (1991). Firm resources and sustained competitive advantage. Journal of Management, 17, 99-120.

Barney, J. B. (2001). Resource-based theories of competitive advantage: a ten-year retrospective on the resource-based view. [Feature]. Journal of Management, 27(6), 643-650.

Barreto, I. (2010). Dynamic Capabilities: A Review of Past Research and an Agenda for the Future. Journal of Management, 36(1), 256-280. doi:10.1177/0149206309350776

Bharadwaj, A., Sambamurthy, V., \& Zmud, R. (1999). IT Capabilities: Theoretical Perspectives and Empirical Operationalization. International Conference on Information Systems (ICIS) ICIS 1999 Proceedings, 378-385.

Bharadwaj, A. S. (2000). A resource-based perspective on information technology capability and firm performance: An empirical investigation. Mis Quarterly, 24(1), 169-196.

BM\&FBOVESPA. (2012). Empresas listadas Retrieved fev de 2012, from http://www.bmfbovespa.com.br/cias-listadas/empresaslistadas/BuscaEmpresaListada.aspx?idioma=pt-br

Dale Stoel, M., \& Muhanna, W. A. (2009). IT capabilities and firm performance: A contingency analysis of the role of industry and IT capability type. [Article]. Information \& Management, 46(3), 181-189. doi:10.1016/j.im.2008.10.002

Field, A. (2009). Descobrindo a estatística usando o SPSS (L. Viali, Trans. 2a ed.). Porto Alegre: Artmed.

Goldoni, V., \& Oliveira, M. (2010). Knowledge management metrics in software development companies in Brazil. [DOI: 10.1108/13673271011032427]. Journal of Knowledge Management, 14(2), 301-313.

Google. (2012). Google Brasil Retrieved Fevereiro de 2012, from http://www.google.com.br/ 
Hartono, E., Li, X., Na, K.-S., \& Simpson, J. T. (2010). The role of the quality of shared information in interorganizational systems use. International Journal of Information Management, 30(5), 399-407. doi:10.1016/j.ijinfomgt.2010.02.007

Huang, S. M., Ou, C. S., Chen, C. M., \& Lin, B. (2006). An empirical study of relationship between IT investment and firm performance: A resource-based perspective. [Article]. European Journal of Operational Research, 173(3), 984-999. doi:10.1016/j.ejor.2005.06.013

ItWeb. (2009-2011). InformationWeek Brasil Retrieved 06 de fev. de 2012, from http://informationweek.itweb.com.br/revista/edicao-241-setembro-2011/

Iudícibus, S. d. (2008). Análise de balanços (9 ed.). São Paulo: Atlas.

Kim, G., Shin, B., Kim, K. K., \& Lee, H. G. (2011). IT Capabilities, Process-Oriented Dynamic Capabilities, and Firm Financial Performance. [Article]. Journal of the Association for Information Systems, 12(7), 487-517.

Kim, J. K., Xiang, J. Y., \& Lee, S. (2009). The impact of IT investment on firm performance in China: An empirical investigation of the Chinese electronics industry. Technological Forecasting and Social Change, 76(5), 678-687. doi:10.1016/j.techfore.2008.03.008

Kraaijenbrink, J., Spender, J. C., \& Groen, A. J. (2010). The Resource-Based View: A Review and Assessment of Its Critiques. Journal of Management, 36(1), 349-372. doi:10.1177/0149206309350775

Lahiri, S., Kedia, B. L., \& Mukherjee, D. (2012). The impact of management capability on the resource-performance linkage: Examining Indian outsourcing providers. [Article]. Journal of World Business, 47(1), 145-155. doi:10.1016/j.jwb.2011.02.001

Liu, Y., Lu, H., \& Hu, J. (2008). IT Capability as Moderator Between IT Investment and Firm Performance. Tsinghua Science and Technology, 13(3), 329-336.

Lucht, R. R., Hoppen, N., \& Maçada, A. C. G. (2007). Ampliação do Modelo de Impacto de TI de Torkzadeh e Doll à luz do Processo Decisório e da Segurança da Informação. Paper presented at the XXXI Encontro da ANPAD, Rio de Janeiro.

Masli, A., Richardson, V. J., Sanchez, J. M., \& Smith, R. E. (2011). Returns to IT excellence: Evidence from financial performance around information technology excellence awards. International Journal of Accounting Information Systems, 12(3), 189-205. doi:10.1016/j.accinf.2010.10.001

Matarazzo, D. C. (2003). Análise financeira de balanços: abordagem básica e gerencial (6 $6^{\mathrm{a}}$ ed.). São Paulo: Atlas.

Muhanna, W. A., \& Stoel, M. D. (2010). How do investors value IT? An empirical investigation of the value relevance of IT capability and IT spending across industries. Journal of Information Systems, 24(1), 43-66. 
Nevo, S., \& Wade, M. R. (2008). Development and validation of scales to measure the strategic potential of IT-enabled resources: A resource-based approach. Paper presented at the 41st Annual Hawaii International Conference on System Sciences 2008, HICSS, Big Island, HI.

Park, J. Y., Im, K. S., \& Kim, J. S. (2011). The role of IT human capability in the knowledge transfer process in IT outsourcing context. Information \& Management, 48(1), 53-61. doi:10.1016/j.im.2011.01.001

Qu, W. G., Oh, W., \& Pinsonneault, A. (2010). The strategic value of IT insourcing: an IT-enabled business process perspective. Journal of Strategic Information Systems, 19, 96-108.

Ray, G., Barney, J. B., \& Muhanna, W. A. (2004). Capabilities, business processes, and competitive advantage: Choosing the dependent variable in empirical tests of the resource-based view. Strategic Management Journal, 25(1), 23-37. doi:10.1002/smj.366

Ray, G., Muhanna, W. A., \& Barney, J. B. (2005). Information technology and the performance of the customer service process: A resource-based analysis. [Article]. Mis Quarterly, 29(4), 625-652.

Santhanam, R., \& Hartono, E. (2003). Issues in linking information technology capability to firm performance. [Article]. Mis Quarterly, 27(1), 125-153.

Schwarz, A., Kalika, M., Kefi, H., \& Schwarz, C. (2010). A Dynamic Capabilities Approach to Understanding the Impact of IT-Enabled Businesses Processes and IT-Business Alignment on the Strategic and Operational Performance of the Firm. [Article]. Communications of AIS, 26, 57-84.

Silva, B. A. M. d. (2011). Decisões do uso da tecnologia da informação: um estudo sobre o efeito das capacidades dinâmicas. Doutorado Tese, Fundação Getúlio Vargas, São Paulo.

Stoel, M. D., \& Muhanna, W. A. (2009). IT capabilities and firm performance: A contingency analysis of the role of industry and IT capability type. Information \& Management, 46, 181-189.

Tallon, P. P. (2010). A Service Science Perspective on Strategic Choice, IT, and Performance in U.S. Banking. [Article]. Journal of Management Information Systems, 26(4), 219-252.

Tallon, P. P., \& Kraemer, K. (2007). Fact or Fiction? A Sensemaking Perspective on the Reality Behind Executives' Perceptions of IT Business Value. Journal of Management Information Systems, 24(1), 13-54. doi:10.2753/mis0742-1222240101

Teece, D. J. (2007). Explicating dynamic capabilities: The nature and microfoundations of (sustainable) enterprise performance. Strategic Management Journal, 28(13), 1319-1350. doi:10.1002/smj.640

Teece, D. J., Pisano, G., \& Shuen, A. (1997). Dynamic capabilities and strategic management. Strategic Management Journal, 18(7), 509-533. 
Tian, J., Wang, K. L., Chen, Y., \& Johansson, B. (2010). From IT deployment capabilities to competitive advantage: An exploratory study in China. [Article]. Information Systems Frontiers, 12(3), 239-255. doi:10.1007/s10796-009-9182-z

Ting-Peng, L., Jun-Jer, Y., \& Chih-Chung, L. (2010). A resource-based perspective on information technology and firm performance: a meta analysis. [Article]. Industrial Management \& Data Systems, 110(8), 1138-1158. doi:10.1108/02635571011077807

Wu, F., Yeniyurt, S., Kim, D., \& Cavusgil, S. T. (2006). The impact of information technology on supply chain capabilities and firm performance: A resource-based view. [Article]. Industrial Marketing Management, 35(4), 493-504. doi:10.1016/j.indmarman.2005.05.003

Wu, Z., Huang, Z., \& Wu, B. (2008). IT Capabilities and firm performance: an empirical research from the perspective of organizational decision-making. Paper presented at the Proceedings of the 2008 IEEE ICMIT.

\title{
TECHNOLOGICAL CAPABILITIES AND FIRM PERFORMANCE ON BRAZILIAN ENTERPRISES MOST INNOVATIVE CONCERNING USE OF IT
}

\begin{abstract}
The objective of this study is to identify the (in) existence of a direct association between IT capabilities and performance at the firm level. Are used secondary data of 122 most innovative companies in the use of IT (67 of them are listed at BOVESPA), published in the magazine ranking. The performance indicators are extracted from the firm's financial statements, both on the website of the Stock Exchange and in the corporate sites, and compared with the score of a company's IT capabilities in the ranking - covering a period of three years (2009-2011). Data are analyzed with non parametric test of Mann Whitney, which takes into account the absence of normality. The results confirm that high IT capabilities are dissociated of the best measures of firm performance, pointing to studies that identify the value of IT in the lower levels of the firm, such as business processes and organizational capabilities, which use IT directly. Limitations and research opportunities are presented in the conclusions.
\end{abstract}

Key-words: IT capabilities; Firm performance; Performance at the process level; Resources; Organizational capabilities.

Data do recebimento do artigo: 30/08/2012

Data do aceite de publicação: 15/02/2013

Revista de Administração e Inovação, São Paulo, v. 10, n.1, p. 79-97, Jan./Mar. 2013. 